\title{
Head \& Face Medicine reviewer acknowledgement 2015
}

Thomas Stamm ${ }^{1 *}$, Ulrich Meyer ${ }^{2}$ and Hans Peter Wiesmann ${ }^{3}$

\section{Contributing reviewers}

The editors of Head \& Face Medicine would like to thank all reviewers who have contributed their time and expertise to the journal in Volume 11 (2015).

\author{
Swetha Acharya \\ India
}

Mehmet Cemal Akay
Turkey

Mohammad Khursheed Alam

Bangladesh

Carlos Eduardo Alcântara

Brazil

\section{E A Al-Moraissi \\ Yemen}

Isabel Andia

Spain

Thaschawee Arkachaisri

Singapore

Wolfgang H. Arnold

Germany

Athanasios Athanasiou

United Arab Emirates

Erica Avila

United States

\section{Alberto Baldini \\ Italy}

Boulos Bechara

United States

Maja Bendyk-Szeffer

Poland

\author{
Darpan Bhargava \\ India \\ Indraneel Bhattacharyya \\ India
}

Kerstin Bitter

Germany

Paolo Boffano

Italy

Ahmad Burhan

Syria

Sandra Bussadori

Brazil

Halil Çagatay

Turkey

Sirmahan Cakare

Turkey

Bing Cheng

China

Fábio Costa

Brazil

Adriano Crismani

Austria

Till Dammaschke

Germany

Gholamreza Danesh

Germany
Yannicke Dauphin
France

Christopher Davis

United Kingdom

Julio Cesar De Oliveira

Brazil

Luciana De-Marchi

Brazil

Ilze Dobele

Latvia

H El Nahass

Egypt

Aala Emara

Egypt

Seyda Ersahan

Turkey

Ahmad Eweida

Egypt

Ali Farahani

United Kingdom

Marijana Filipovska-Musanovic Bosnia And Herzegovina

Roman Fischbach

Germany

Patrice Forget

Belgium

* Correspondence: headfacemed@biomedcentral.com

${ }^{1}$ University of Münster, Münster, Germany

Full list of author information is available at the end of the article 
Edward Franek

Poland

Roland Frankenberger

Germany

Bernhard Frerich

Germany

Andreas R. Gantenbein

Switzerland

Robert Garcia

France

Olaide S Gbadebo

Nigeria

R Gedik

Turkey

Mehdi Gholami

Iran

Merve Goymen

Turkey

Chuanbin Guo

China

Yi Guo

United States

Laurent Guyot

France

Urban Hagg

Hong Kong

Mohammad Hajeer

Syria

Xin-Wei Han

China

K V S Hari Kumar

India

Ayman Hegab

Egypt

Andrew Hinson

United States

Ariane Hohoff

Germany

Akihiro Hosoya

Japan

Kazuhiko Imaizumi

Japan

Belma Isik Aslan

Turkey
Mario Isiordia

Mexico

Eren Isman

Turkey

Yusuf Izci

Turkey

Gundega Jakobsone

Latvia

Xin-Chun Jian

United States

Susanne Jung

Germany

Britta A. Jung

Germany

P Jungbluth

Denmark

Graça Justo

Brazil

Joseph Katz

United States

Hong-Seop Kho

South Korea

Tae-Woo Kim

South Korea

Gero Kinzinger

Germany

Christian Kirschneck

Germany

Michael Knoesel

Germany

Doruk Kocyigit

Turkey

Heike Korbmacher-Steiner

Germany

Fatih Mehmet Korkmaz

Turkey

Jolanta Kostrzewa-Janicka

Poland

Gerald Krennmair

Antarctica

Tae-Geon Kwon

South Korea

Manuel O Lagravere

Canada
Evangeli S Lampri

Greece

Rainer Laskawi

Germany

Guenter Lauer

Germany

Uilyong Lee

South Korea

Carsten Lippold

Germany

Di-Qing Luo

China

Robert Bruce Macintosh

Micronesia

Manuele Mancini

Italy

Giuditta Mannelli

Italy

Peter Maurer

Germany

Edward Mbewe

Zambia

Margaret Mclaughlin-Drubin

United States

Francisco Javier Medina Fernández Spain

Giuseppe Monaco

Italy

Urs D. A. Müller-Richter

Germany

Takahito Nakajima

Japan

Jörg Neunzehn

Germany

Ruba Odeh

United Kingdom

Fatih Oghan

Turkey

Jooyoung Ohe

South Korea

Sergio Olate

Chile

Michelle Ommerborn

Germany 
Siti Adibah Othman

Malaysia

Mario Pagnoni

Italy

Vitor Panhóca

Brazil

Chan Hum Park

South Korea

Jae Park

United States

Anne-Lise Poirrier

Belgium

Michal Polguj

Poland

Amaury Pozos

Mexico

Vahid Rakhshan

Iran

Ksenija Rener-Sitar

Slovenia

Michael Richards

United States

Matthias Roggendorf

Germany

Jianping Ruan

China

Parappa Sajjan

India

Atsushi Sano

Japan

Kes Schroer

United States

Matthias Schulz

Germany
Falk Schwendicke

Germany

Robin Seeberger

Germany

Ahmet-Ercan Sekerci

Turkey

Emmanuel Joao Nogueira Leal Silva

Brazil

Paulo Goberlanio De Barros Silva

Brazil

Ralf Smeets

Germany

Janet Southerland

United States

Derek Steinbacher

United States

Markus Stenner

Germany

Lisha Sun

China

Enes Tan

Turkey

Bien Keem Tan

Singapore

Ichih Tan

United States

Der-Cherng Tarng

Taiwan

Umut Tekin

Turkey

Afshin Teymoortash

Germany

Weidong Tian

China
Ji Tong

China

Florian Uecker

Germany

Jop Verweij

Netherlands

Joan Viciano

Italy

Rick Visser

Spain

J Von Bremen

Germany

Wen-Hung Wang

Taiwan

Nuray Yilmaz Altintas

Turkey

Shengyuan Yu

China

Yehuda Zadik

Israel

Mohammad Zandi

Iran

Yu Zhou

China

Thomas Ziebura

Germany

Author details

University of Münster, Münster, Germany.

${ }^{2}$ University of Düsseldorf, Düsseldorf, Germany.

${ }^{3}$ Technische Universität Dresden, Dresden,

Germany. 